\title{
14-in Diagonal Size Nanotip-Enhanced Plasma Lamp in Liquid Crystal Display
}

\author{
Po-Chuan Tsai and Sung-O Kim, Member, IEEE
}

\begin{abstract}
The characteristics of nanotip-enhanced plasma lamps (NTL) as a backlight for liquid crystal displays have been investigated on a 14-in panel. Using a direct-current pulse bipolar waveform driving, the NTL operates with lower ignition and sustain voltages below $300 \mathrm{~V}$ compared with conventional plasma lamps due to the tiny discharge gap between the indium tin oxide electrode and nanotip electrodes. Luminous efficiencies of $16 \mathrm{~lm} / \mathrm{W}$ at the lowest ignition voltage of $150 \mathrm{~V}$ have been realized for NTL, which correspond to $3780 \mathrm{~cd} / \mathrm{m}^{2}$ of luminance in $260 \mathrm{~V}$ with $50 \mathrm{torr}$ of pure xenon gas.
\end{abstract}

Index Terms-Lamps, LCD, microdischarge.

$\mathbf{R}$ ECENTLY, backlighting liquid crystal display (LCD) panels has become a key research issue for increasing the diagonal size for monitor to television applications. In increasing the diagonal size of the panel, cost is a major factor. Light-emitting diode backlight is still expensive compared with other backlights such as cold cathode fluorescent lamp, hot cathode fluorescent lamp, external electrode fluorescent lamp, and flat fluorescent lamp. Furthermore, the driving voltage of the existing backlights is higher than the $1-2 \mathrm{kV}$ required to operate LCD. Producing difficulties in terms of luminous efficiency, cost, and electrical hazard. In this paper, we report on the nanotip-enhanced plasma lamp (NTL) using nanotip electrodes which are fabricated by semiconductor processes. The NTL ignites a wide and stable plasma along the indium tin oxide (ITO) electrode and silver electrodes on the front and rear panels, respectively [1]. The NTL excited the phosphor layer by vacuum ultraviolet (VUV) and generated visible white light at a lower ignition voltage because of the field emission at the nanotip electrodes. A plasma lamp has been reported, which used carbon nanotube as a needle electrode to converge the equipotential lines to improve the local electric field and generate the field emission [2]. The NTL uses xenon as a discharge gas to replace mercury. We investigated a 14-in NTL mercury free flat plasma lamp for LCD backlight with low driving voltage, high luminance, and luminous efficiency.

A schematic diagram of the NTL is shown in Fig. 1. The NTL consisted of two parts: the front panel, and the rear panel.

Manuscript received November 19, 2007; revised March 11, 2008. This work was supported in part by the Technology Development Program for Academia, Ministry of Economic Affairs (MOEA) in Taiwan (96-EC-17-A-07-S1-046), by the National Science Council (NSC 96-2221-E-009-079-MY3), by ChungHwa Picture Tubes, Ltd. (N96-06), and by the Semiconductor Research Corporation.

The authors are with the Department of Photonics and Display Institute National Chiao Tung University, Hsin-Chu City 30010, Taiwan, R.O.C. (e-mail: anthony.di95g@nctu.edu.tw; sostar@mail.nctu.edu.tw).

Color versions of one or more of the figures in this paper are available online at http://ieeexplore.iee.org.

Digital Object Identifier 10.1109/TPS.2008.922942

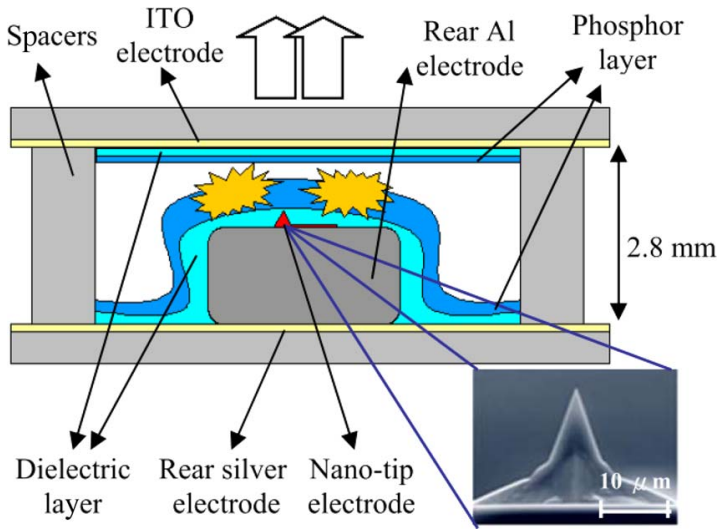

Fig. 1. Cross-sectional schematic diagram of NTL.

A high distortion point glass (Asahi glass, PD-200) is used as substrates in the front and rear panels. The size of the glass substrate is $320 \times 300 \mathrm{~mm}$. The silver electrodes have been deposited on the rear glass substrate by a screen printing method. Then, the nanotip electrodes on the aluminum rods were fabricated on the silver electrodes. The ITO electrode is patterned by conventional photolithographic techniques and wet etching method on the front panel. The dielectric and phosphor layers are deposited on the electrodes at the rear panel and on the ITO electrode at the front panel, respectively. Finally, both panels are sealed by frames and spacers which are $2.8 \mathrm{~mm}$ in height in order to maintain the effective discharge volume. The NTL is evacuated to $10^{-6}$ torr using a rotary and turbo molecule pump, and backfilled with research vacuum grade pure xenon gas, emitting 147- and 173-nm photons from the excited xenon monomer and dimer, respectively. The NTL is driven by a power supply (Shen Chang Electric CO Ltd., SPIK 2000A) which provided direct-current pulse bipolar waveform at $50 \mathrm{kHz}$.

Fig. 2 shows the plasma with $260 \mathrm{~V}$ in 50 torr of pure xenon gas. The operating voltages without nanotips were more than $1 \mathrm{kV}$. The discharge current parabolically increased with applied voltage. The plasma resistance of the NTL was positive. The luminance and luminous efficiency of the NTL were $1250-3780 \mathrm{~cd} / \mathrm{m}^{2}$ and $16-11 \mathrm{~lm} / \mathrm{W}$, respectively, as a function of the voltage in the range of 150-260 V. The luminance of the NTL improved as a function of the applied voltage, whereas the luminous efficiency decreased because additional power is used to expand the plasma from the sharp nanotip to a more flat electrode. The nanotip electrodes provide field emission to reduce the breakdown voltage.

In summary, the NTL provides a plasma with high luminous efficiency of $16 \mathrm{~lm} / \mathrm{W}$ at the lowest ignition voltage of $150 \mathrm{~V}$. 


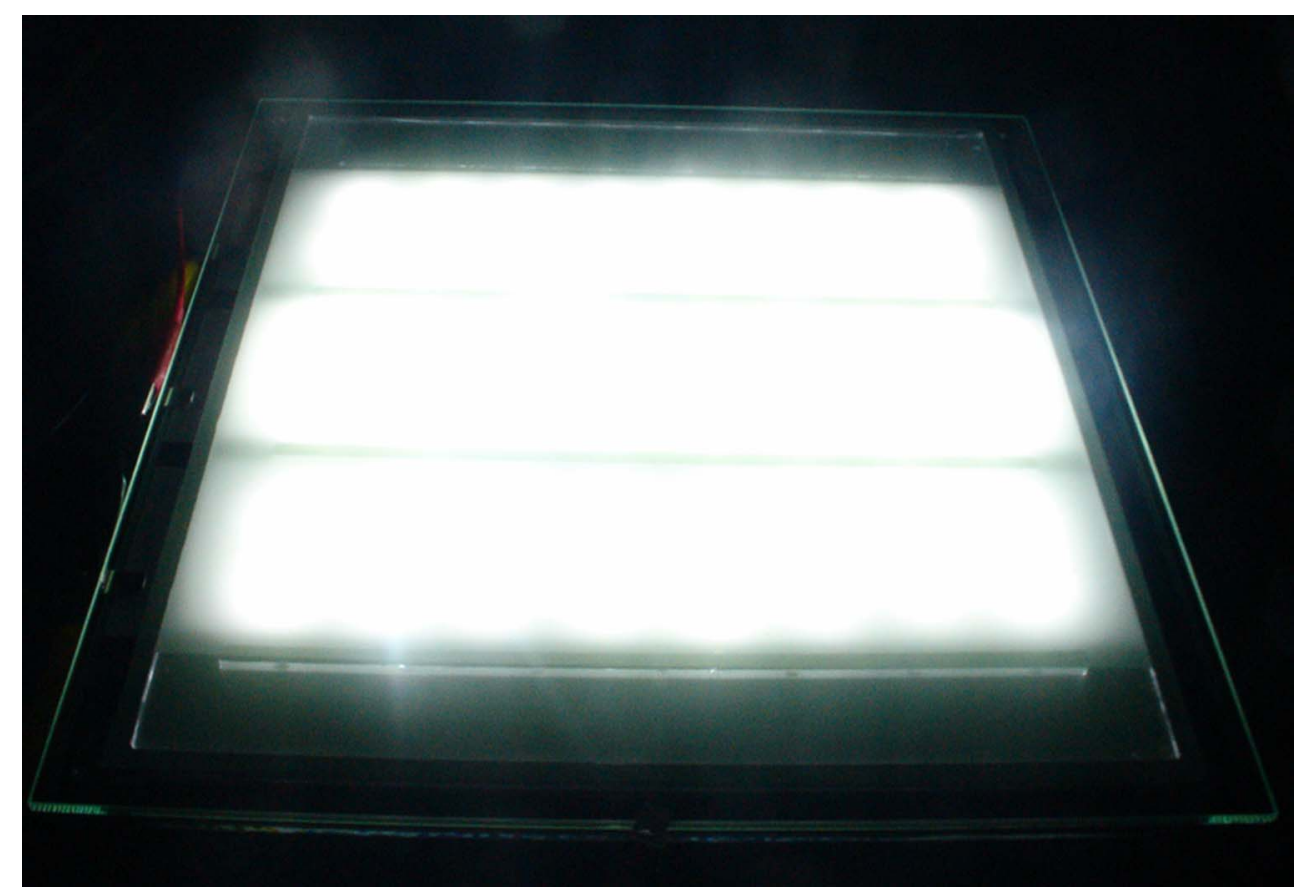

Fig. 2. Photograph of the 14-in NTL while igniting the plasma at $260 \mathrm{~V}$ in 50 torr of pure xenon gas.

The luminance and luminous efficiency were $3780 \mathrm{~cd} / \mathrm{m}^{2}$ and $11 \mathrm{~lm} / \mathrm{W}$ in $260 \mathrm{~V}$, respectively. The NTL has successfully provided a bright, uniform, and stable plasma in 14-in diagonal panel as a backlight for LCD.

\section{ACKNOWLEDGMENT}

The authors would like to thank Dr. C.-N. Mo and S.-C. Wu, as well as colleagues in nanosystem and plasma laboratory, for their support.

\section{REFERENCES}

[1] S.-O. Kim and J. G. Eden, "Arrays of square cross-section microdischarge devices fabricated in glass and driven by interdigitated electrodes," IEEE Trans. Plasma Sci., vol. 33, no. 2, pp. 566-567, Apr. 2005.

[2] J. T. H. Tsai and H. C. Ko, "Plasma illumination devices enhanced by carbon nanotubes," Appl. Phys. Lett., vol. 88, no. 1, p. 013104 , Jan. 2006. 